\title{
Papers
}

\section{Randomised controlled trial of effects of early discharge after surgery for breast cancer}

Nigel Bundred, Peter Maguire, Jill Reynolds, Jill Grimshaw, Julie Morris, Lesley Thomson, Lester Barr, Andrew Baildam

\begin{abstract}
Objective To determine the effect of early discharge from hospital after surgery for breast cancer on physical and psychological illness.

Design Randomised controlled trial comparing discharge two days after surgery (before removal of drain) with standard management (discharge after removal of drain).

Setting Regional breast unit.

Subjects 100 women with early breast cancer undergoing mastectomy and axillary node clearance (20) or breast conservation surgery (80).

Main outcome measures Physical illness (infection, seroma formation, shoulder movement) and psychological illness (checklist of concerns, Rotterdam symptom questionnaire, hospital anxiety and depression scale) preoperatively and at one month and three months postoperatively.

Results Women discharged early had greater shoulder movement (odds ratio 0.28 (95\% confidence interval 0.08 to 0.95$) ; \mathrm{P}=0.042$ ) and less wound pain (odds ratio 0.28 (0.10 to 0.79$) ; \mathrm{P}=0.016$ ) three months after surgery compared with women given standard management. One month after surgery scores were significantly lower on the Rotterdam symptom questionnaire in patients who were discharged early (ratio of geometric mean scores 0.73 (0.55 to 0.98$) ; \mathrm{P}=0.035)$, but rates of psychological illness generally did not differ between groups. Conclusions Increased rates of physical or psychological illness did not result from early discharge after surgery for breast cancer. This policy can be recommended for patients with support at home.
\end{abstract}

\section{Introduction}

Increasing hospital costs have raised demands for improved efficiency in the use of hospital beds. Early discharge of patients who have had surgery for breast cancer is routine practice in the United States, but the average postoperative stay in the United Kingdom is seven days. ${ }^{1-5}$

Physical recovery after surgery is rapid, but patients require the insertion of drains to prevent fluid collecting in their wounds (seroma formation) and usually remain in hospital until the drains are removed. Early removal of drains leads to an unacceptably high rate of seromas, which often require repeated drainage by needle aspiration in outpatient clinics..$^{1-5}$ Other potential complications of early discharge include an increased rate of wound infection and decreased shoulder function, ${ }^{1-4}$ although an open nonrandomised study reported no increase in complications. ${ }^{5}$ Another potential benefit from extended stay is that it may aid psychological adaptation to the cancer diagnosis and changed physical appearance.

Several non-randomised studies in the United States have asserted that the stay in hospital for patients with breast cancer can be safely reduced by five days if women are discharged with wound drains still in place and have support at home from a partner..$^{2-4}$ Cohen et al compared 39 patients who had been discharged early with historical controls and reported that average stay in hospital was reduced from 9.5 to 4.7 days. ${ }^{3}$ Edwards et al reported an average reduction of six days in hospital in 61 patients and claimed a $39 \%$ reduction in hospital costs. ${ }^{4}$ If a similar reduction of 5-6 days' stay could be achieved in the United Kingdom hospital costs would be reduced by around $£ 1000$ per patient (five days in hospital at $£ 200$ a day), yielding potential savings to the NHS.

Despite the widespread practice of discharging patients 48 hours after surgery in the United States, no studies have compared this practice with standard British practice. Management of women after breast surgery in the United Kingdom has remained hospital based because of an unwillingness to redeploy hospital based specialist breast nurses to outpatient clinics $^{5}$ and concerns that early discharge might have adverse psychological effects. Moreover, the impact of discharge within three days on physical or psychological illness in patients or on workload in primary care has not, to our knowledge, been assessed in a randomised controlled trials to date.

We tested the hypothesis that early discharge after surgery for cancer has no adverse physical or psychological effects on patients.

\section{Patients and methods}

From 1 January 1996 to 1 April 1997, 365 women undergoing surgery for early breast cancer in this breast unit were offered either early discharge at two days after surgery (with an axillary drain still in place) or standard discharge after all drains have been

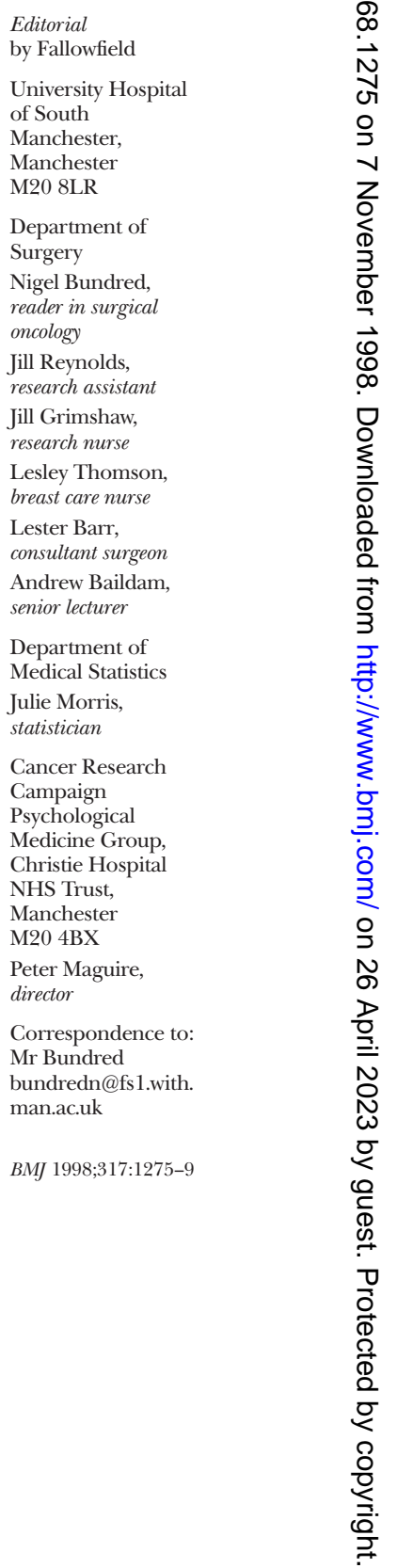




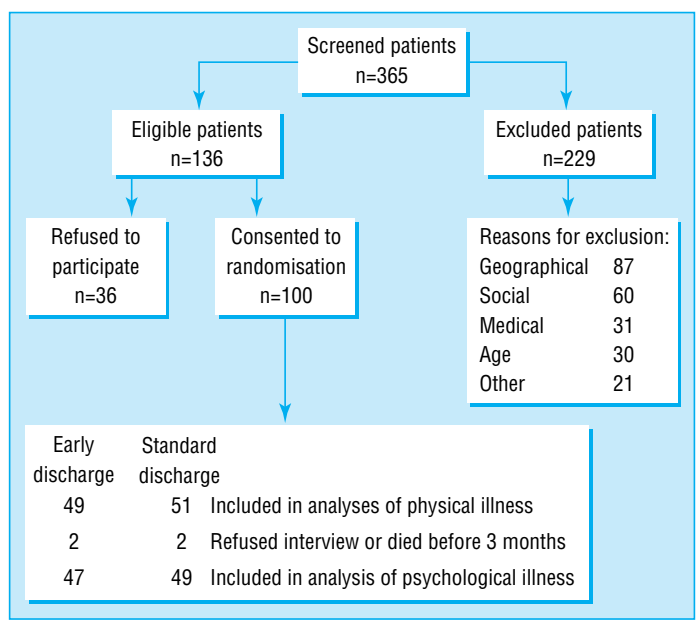

Inclusion of patients in study

removed as part of a randomised controlled trial. The trial was approved by the South Manchester Ethics Committee, and all women gave written informed consent. Thirty women were excluded from the trial because they were over $75 ; 87$ because they lived in health districts more than $32 \mathrm{~km}$ from this hospital; 60 because they had no social support at home; 31 because they had concurrent medical illness; and 21 for other reasons such as language difficulties. Of the 136 eligible patients, 100 consented to the trial and 36 refused (figure). We aimed to recruit 200 women to also assess care by district nurses of patients discharged early, but in this paper we report the results of a planned interim analysis to confirm no adverse consequences of early discharge in the first 100 women to complete the study.

Patients who consented to the trial were recruited in an outpatient clinic and given an admission date (usually 2-3 weeks later). At the beginning of the week of each admission a sealed envelope was opened by a research nurse (JG) to find the randomisation code for that week (either standard discharge or early discharge). Patients were thus randomised in clusters by week. The number of patients admitted during a week varied from one to four, with an average of two. To ensure that early discharge had no adverse consequences we required a sample size of around 50 patients in each group. Given an average cluster size of two and an intraclass correlation coefficient of 0.02 , the study would have $80 \%$ power to test the equivalence of outcomes to within $20 \%$.

Women were treated by three consultant surgeons $(\mathrm{NB}, \mathrm{LB}$, and $\mathrm{AB})$, all of whom adopted an agreed discharge policy. Patients randomly allocated to early discharge were sent home 48 hours postoperatively with an axillary siphon drain in place. Standard management consisted of keeping the patients in hospital until wound drainage was less than $50 \mathrm{ml} /$ day, when the drain could be removed (usually days 5-10).

Patients randomly allocated to early discharge were instructed how to manage the wound drain and given information sheets on wound care. Their partners were also given instruction and information sheets. They were asked to measure the volume of fluid draining from the wound and were telephoned daily by specialist breast nurses and visited by them every other day. A
24 hour telephone contact number was provided during the study for all patients. The drain was removed when the volume of fluid was less than $50 \mathrm{ml}$ a day. Breast nurses also communicated with district nurses about the management of wound drainage in patients with breast cancer.

\section{Study end points}

The amount of fluid drained and the time to drain removal was compared for each group, as well as wound complications, including haematoma, seroma, and infection (defined as any redness of the wound or any episodes requiring antibiotic treatment). The number of return visits to hospital and any extra visits to see general practitioners or district nurses were recorded in each group.

Shoulder movement was assessed preoperatively and at one and three months postoperatively in both groups by the research nurse (JG). Patients were asked about functional shoulder movements, and the degree of abduction, adduction, internal or external rotation, flexion, and extension was measured. In particular, any limitation of movement that prevented a patient combing her hair or fastening her bra was noted. Both groups were visited by a physiotherapist before discharge to advise on shoulder exercises.

All patients were asked to record how many times they visited their district nurse or general practitioner, and their requirement for analgesia was used to define the presence or absence of wound pain.

The hospital anxiety and depression scale, Rotterdam symptom checklist, and Spielberger state or trait anxiety inventory were completed by the patients preoperatively and at one and three months postoperatively to measure their mood state, current concerns, and quality of life. ${ }^{6-8}$ A checklist of concerns was developed from work by Devlen. ${ }^{9}$

\section{Statistical analysis}

Data were analysed on the basis of intention to treat using the statistical package stATA. To assess the significance of differences between the groups we used logistic regression analysis with adjustment for clustering on weeks for binary outcomes such as wound pain and number of visits to a general practitioner. Results are expressed as odds ratios with 95\% confidence intervals. For continuous outcomes such as psychiatric illness and time until removal of the drain we used random effects linear regression analysis with adjustment for clustering on weeks. These data were all positively skewed and required logarithmic transformation to achieve a reasonable approximation to a normal distribution. Results are expressed as geometric means and ratios of mean effects with $95 \%$ confidence intervals.

\section{Results}

Data on physical illness were collected from all of the 136 eligible patients. Data on psychiatric illness were collected only from patients who agreed to the study. As two women died three months after the operation and two refused to be interviewed, the psychiatric outcome is based on data from 96 women (47 allocated to early discharge and 49 to standard discharge) (figure). 
Table 1 Physical illness among women after surgery for breast cancer. Values are numbers (percentages) of patients unless stated otherwise

\begin{tabular}{|c|c|c|c|c|}
\hline & $\begin{array}{l}\text { Early discharge } \\
\qquad(\mathrm{n}=49)\end{array}$ & $\begin{array}{c}\text { Standard } \\
\text { discharge }(n=51)\end{array}$ & $\begin{array}{l}\text { Ratio or odds ratio } \\
(95 \% \mathrm{Cl})^{*}\end{array}$ & $P$ valuet \\
\hline Geometric mean time to removal of drain (range) (days) & $5.5(3-11)$ & $5.3(2-12)$ & $1.02(0.89$ to 1.18$)$ & 0.74 \\
\hline Geometric mean No of aspirations required (range)‡ & $1.9(1-9)$ & $2.2(1-4)$ & $0.86(0.58$ to 1.28$)$ & 0.46 \\
\hline Readmission & $1(2)$ & $3(6)$ & 0.33 (0.03 to 3.19$)$ & 0.34 \\
\hline Seroma formation & $17(36)$ & $19(40)$ & $0.86(0.38$ to 1.95$)$ & 0.73 \\
\hline \multicolumn{5}{|l|}{ At 3 months: } \\
\hline Wound infection & $12(26)$ & $16(33)$ & $0.69(0.28$ to 1.66$)$ & 0.40 \\
\hline Arm or hand swelling & $3(6)$ & $7(15)$ & $0.39(0.10$ to 1.44$)$ & 0.16 \\
\hline Restricted shoulder movement & $5(11)$ & $14(30)$ & $0.28(0.08$ to 0.95$)$ & 0.042 \\
\hline Wound pain & $7(15)$ & $18(39)$ & $0.28(0.10$ to 0.79$)$ & 0.016 \\
\hline
\end{tabular}

${ }^{\star}$ Ratio of early to standard discharge or with standard discharge as reference group, both adjusted for clustering on weeks.

†For differences between patients allocated to early discharge and those allocated to standard discharge.

fFor patients requiring aspiration (17 allocated to early discharge, 19 allocated to standard discharge).

\section{Physical outcome}

The median age of women allocated to early discharge was 54 (range 31-74) compared with 57 (38-74) in those allocated to standard discharge. Eighty per cent of each group had a partner at home and 20\% had other support. Eleven of the 36 women who refused to participate $(31 \%)$ were scheduled for mastectomy compared with 16 of the group who consented to the trial $(16 \%)$. The median hospital stay postoperatively in the patients who were discharged early was $2(2-8)$ days compared with 5 (3-13) days in the patients who were discharged in the standard time, and 6 (1-11) in those who refused to participate in the trial. In total, 42 patients allocated to early discharge had an axillary drain still in place three days postoperatively. Five patients allocated to early discharge remained in hospital because of medical problems or home circumstances. One patient allocated to early discharge required readmission to hospital three weeks after surgery because of a pulmonary embolism (which developed after starting chemotherapy) and another in the same group died of a myocardial infarction seven days postoperatively without having been discharged. Three patients allocated to standard discharge were readmitted to hospital with wound infections (table 1).

No difference was observed in rates of infection, seroma formation, or arm swelling. Shoulder movement three months postoperatively was better in the women discharged early, with less restriction of movement (odds ratio 0.28 (95\% confidence interval 0.08 to 0.95)). The women discharged early also had less wound pain because they required less analgesia (odds ratio 0.28 (0.10 to 0.79$)$ ) (table 1 ).

In the first month after discharge the number of visits to general practitioners did not differ between groups (23 in women allocated to standard discharge $v$ 20 in those allocated to early discharge), but by the third month more patients allocated to standard discharge had consulted their family doctor (15 $v 9$, $\mathrm{P}=0.20)$. No difference in the number of consultations with nurses was seen. Patients' visits to their family doctors during the second and third months were significantly related to reduced shoulder movement $(\mathrm{P}=0.042)$ and increased wound pain $(\mathrm{P}=0.016)$.

\section{Psychological outcome}

Table 2 shows the geometric mean scores for questionnaires in the two groups. Scores on the concerns checklist, hospital anxiety and depression scale, and Spielberger questionnaire were similar in the two groups. The group allocated to standard discharge scored significantly higher on the Rotterdam symptom questionnaire at one month $(\mathrm{P}=0.035)$, but this difference may be a chance finding due to the large number of psychological scores compared.

The number in each group who scored as cases-that is, had scores above the cut off point for risk of psychiatric disorder-on the concerns checklist or hospital anxiety and depression scale was assessed preoperatively. A higher proportion of the women allocated to early discharge scored as cases on both measures $(24(51 \%) v 19(39 \%))$, although the number of cases fell by one month in the women allocated to early discharge but not in the other women $(12(25 \%) v$ $19(39 \%))$. This difference had equalised for both groups by three months after surgery. A trend for women in the early discharge group to show greater concern preoperatively (probably because of initial anxieties about the prospect of early discharge) was balanced by a noticeable reduction in psychological distress by one month postoperatively.

Table 2 Geometric mean scores (ranges) on questionnaires assessing psychological illness among women after surgery for breast cancer

\begin{tabular}{lcccc} 
Questionnaire & Early discharge & Standard discharge & Ratio* $(95 \%$ Cl) & P value \\
\hline Preoperatively & & & & \\
\hline Concerns checklist & $5.9(0-25)$ & $5.8(0-32)$ & $1.00(0.76$ to 1.32$)$ & 0.97 \\
\hline Anxiety & $3.9(0-21)$ & $3.4(0-19)$ & $1.10(0.76$ to 1.57$)$ & 0.63 \\
\hline Depression & $1.6(0-15)$ & $1.6(0-15)$ & $1.00(0.72$ to 1.40$)$ & 0.99 \\
\hline Hospital anxiety and depression & $5.7(0-36)$ & $5.1(0-34)$ & $1.07(0.72$ to 1.60$)$ & 0.73 \\
\hline Rotterdam & $8.1(0-40)$ & $9.4(0-51)$ & $0.87(0.59$ to 1.27$)$ & 0.47 \\
\hline Spielberger & $37.2(20-73)$ & $37.1(21-77)$ & $1.00(0.86$ to 1.15$)$ & 0.92 \\
\hline At 1 month & & & \\
\hline Concerns checklist & $4.4(0-22)$ & $5.0(0-28)$ & $0.92(0.66$ to 1.30$)$ & 0.65 \\
\hline Anxiety & $1.7(0-13)$ & $1.7(0-10)$ & $1.00(0.69$ to 1.45$)$ & 0.99 \\
\hline Depression & $1.6(0-20)$ & $2.4(0-18)$ & $0.76(0.56$ to 1.03$)$ & 0.076 \\
\hline Hospital anxiety and depression & $3.3(0-33)$ & $4.1(0-25)$ & $0.84(0.57$ to 1.24$)$ & 0.38 \\
\hline Rotterdam & $7.0(0-54)$ & $10.0(1-51)$ & $0.73(0.55$ to 0.98$)$ & 0.035 \\
\hline Spielberger & $29.2(20-65)$ & $31.2(20-71)$ & $0.94(0.82$ to 1.07$)$ & 0.35 \\
\hline At $\mathbf{3}$ months & & & & \\
\hline Concerns checklist & $3.7(0-19)$ & $2.8(0-36)$ & $1.23(0.90$ to 1.69$)$ & 0.20 \\
\hline Anxiety & $1.4(0-10)$ & $1.7(0-17)$ & $0.92(0.69$ to 1.23$)$ & 0.58 \\
\hline Depression & $1.2(0-11)$ & $1.7(0-15)$ & $0.80(0.60$ to 1.08$)$ & 0.14 \\
\hline Hospital anxiety and depression & $2.3(0-21)$ & $3.5(0-32)$ & $0.77(0.57$ to 1.05$)$ & 0.097 \\
\hline Rotterdam & $6.3(0-60)$ & $7.0(0-57)$ & $0.87(0.66$ to 1.13$)$ & 0.32 \\
\hline Spielberger & $28.7(20-71)$ & $28.7(20-76)$ & $1.00(0.90$ to 1.12$)$ & 0.99 \\
\hline Early to standar & & &
\end{tabular}

*Early to standard discharge adjusted for clustering on weeks. 
Questionnaires on patient satisfaction showed that all patients allocated to early discharge and 98\% of those allocated to standard discharge thought that they received sufficient support at home. Ninety per cent of patients allocated to early discharge and $77 \%$ of those allocated to standard discharge were very pleased with the care given by the breast care nurse.

\section{Discussion}

To reduce the costs of health care in the United States patients undergoing mastectomy are discharged routinely 24 hours after surgery. Although palliative care for patients with cancer is widely available at home in parts of Europe $\mathrm{e}^{10}$ and home care is the fastest growing component of health care in the United States, ${ }^{11}$ few randomised trials assessing its effect on rates of illness have been carried out.

In our controlled trial women were randomly allocated to each discharge policy and a reduction in length of hospital stay was achieved without any increase in rates of illness, showing that early discharge should be used more often after surgery for breast cancer. The women discharged earlier had lower rates of illness postoperatively. The greater contact with general practitioners by patients allocated to standard discharge was a result of a higher incidence of physical illness (reduced shoulder movements and increased wound pain) that required more analgesia.

In the case of patients undergoing breast conservation who require radiotherapy the increased incidence of restricted shoulder movement in women allocated to standard management (but not early discharge) may lead to a delay in starting radiotherapy because shoulder movement must be optimised before treatment can be started.

Up to $25 \%$ of women develop a depressive illness after mastectomy. ${ }^{8}$ Psychological illness was not affected by early discharge, but women thought that being discharged early boosted their confidence and increased their sense of reassurance.

Enthusiasm for early discharge should be tempered by the fact that $27 \%$ of women were ineligible for early discharge because of geographical factors. This relates to our breast unit in particular, which treats women throughout greater Manchester. The effect of geographical factors is being considered in the second year of our study, in which care by district nurses is being evaluated to enable more women to be included. The study also continues to allow assessment of economic benefits, and our preliminary data suggest that care from district nurses (rather than from breast care nurses in hospitals) does not affect outcome. In addition, increased numbers of women undergoing early discharge after mastectomy will allow confirmation of the safety of this policy.

Early discharge is possible only when careful attention is paid to perioperative care and to instruction of patients and families on managing the drain. It also requires extra input from nurses to gain patients' confidence in the policy, ${ }^{4}$ but it reduced the need for medical intervention by general practitioners.

The average length of stay has fallen by 34 days over the past 40 years. Although Bonnema et al recently claimed that early discharge was beneficial, they investigated discharge four days postoperatively, ${ }^{12}$
- The average hospital stay after surgery for breast cancer is seven days in the United Kingdom

- This study found that discharge two days after such surgery does not affect rate of complications

- A short stay in hospital with support from specialist breast care nurses on the patient's return home is acceptable to patients

- Psychosocial illness is not affected by early discharge

- Early discharge home facilitates shoulder movement and reduces subsequent wound pain

- Patients suitable for early discharge must have support from a relative at home and be in good physical health

which is already practised in the United Kingdom. ${ }^{15}$ We have confirmed the benefits of discharge two days postoperatively. If translated into management policy throughout the United Kingdom early discharge could provide substantial savings. Our study also includes economic appraisal of the costs (to the NHS and patients), and the results will be available when the second year of the study is completed. Nevertheless, these preliminary results show that early discharge is safe and has no adverse effects on physical or psychological illness after surgery for breast cancer.

Our results support a reduction in the length of hospital stay for patients with breast cancer and suggest that home care provides superior clinical outcomes with greater patient satisfaction.

We thank all the ward nurses and breast care nurses who helped with the study, and Mrs M Gaskell for secretarial help.

Contributors: NB contributed to the design of the protocol, performed a literature search, participated in the execution of the study, collected and analysed the data, wrote the paper, and is guarantor for the study. JR coordinated the study at both hospitals, discussed core ideas, studied the literature, participated in data collection and analysis, and contributed to the writing of the paper. JG and LT provided the specialist breast care nursing, recruited the patients, and provided the structured information about early discharge. JM carried out the statistical analysis. PM coordinated the psychosocial assessment of the patients in the study, contributed to the design of the psychosocial protocol, interpreted the data, and helped with writing the paper. LB and $\mathrm{AB}$ participated in the collection of the data, contributed to the interpretation of the findings, and helped with drafting the article. All authors approved the final version of the paper to be published.

Funding: This study was funded by the NHS national research and development programme (grant No 16-05). We thank Simcare Health Products for providing the siphon drains. Conflict of interest: None.

1 Ball ABS, Fish S, Waters R, Merrion Thomas J. Radical axillary dissection in the staging and treatment of breast cancer. Ann R Coll Surg Engl 1992;74:126-9.

2 Funnell IC, Crowe PJ, Dent DM. Does surgical experience influence mastectomy complications? Ann R Coll Surg Engl 1992;4:178-80.

3 Cohen AM, Schaeffer N, Chen Z, Wood WC. Early discharge after modified radical mastectomy. Am J Surg 1986;15:465-6.

4 Edwards MJ, Broadwater RJ, Bell JL, Ames MD, Balch CM. Economic impact of reducing hospitalisation for mastectomy patients. Ann Surg 1988;208:330-6.

5 Holcombe C, West N, Mansel RE, Horgan K. The satisfaction and savings of early discharge with drain in situ following axillary lymphadenopathy in the treatment of breast cancer. Eur J Surg Oncol 1995;21:604-6. 
6 De Haes JCJM, van Knippenberg FCE, Neijt JP. Measuring psychological and physical distress in cancer patients; structure and application of the Rotterdam symptom checklist. Br J Cancer 1990;62:1034-8.

7 Ibbotson T, Maguire P, Selby P, Priestman T, Wallace L. Screening for anxiety and depression in cancer patients: the effects of disease and treatment Eur J Cancer 1994.30A.37-40.

8 Fallowfield LJ, Hall A, Maguire GP, Baum M. Psychological outcomes of different treatment policies in women with early breast cancer outside a different treatment policies in women
clinical trial. $B M J$ 1990;301:575-80.

clinical trial. $B M J$ 1990;301:575-80.
Devlen J. Psychological and social morbidity in lymphoma patients $[\mathrm{PhD}$

thesis]. Manchester: University of Manchester 1984.
10 Pannuti F, Tannenberger S. The Bologna Eubiosia Project: hospital-athome care for advanced cancer patients. J Palliat Care 1992;8:11-7.

11 Hing E. Characteristics of elderly home health patients: preliminary data from the 1992 national home and hospice care survey. National Center for Health Statistics, 1994

12 Bonnema J, van Wersch AMEA, van Geel AN, Pruyn JFA, Schmitz PIM, Paul MA, et al. Medical and psychosocial effects of early discharge after surgery for breast cancer: randomised trial. BMJ 1998;316:1267-70.

(Accepted 26 June 1998)

\title{
One day survey by the Mental Health Act Commission of acute adult psychiatric inpatient wards in England and Wales
}

\author{
Richard Ford, Graham Durcan, Lesley Warner, Pollyanna Hardy, Matt Muijen
}

\begin{abstract}
Objectives To provide (via the Mental Health Act Commission's "national visit") empirical evidence on ward occupancy levels, use of the Mental Health Act 1983, nurse staffing, and care of female patients on acute adult psychiatric wards.

Design One day survey of a stratified random sample. Settings 119/250 (47\%) acute adult psychiatric inpatient units in England and Wales.

Subjects End sample of 263 acute psychiatric inpatient wards.

Main outcome measures Ward occupancy rates; number of patients detained under the Mental Health Act and proportion "absent without leave"; nurse staffing levels, skill mix, and vacancies; proportion of women with self contained, women-only facilities.

Results Mean ward occupancy was 99\% (95\% confidence interval $97 \%$ to $102 \%$ ). A ward mean of $30 \%(28 \%$ to $32 \%)$ of patients were detained under the Mental Health Act; of all detained patients, $1 \%$ ( $1 \%$ to $2 \%)$ were absent without leave. A ward mean of $0.3(0.29$ to 0.31$)$ nurses were on duty per patient at the time of the visit. An estimated ward mean of $31 \%$ $(30 \%$ to $32 \%)$ of nurse staffing may have been through casual contracts-higher in inner (48\% (43\% to $53 \%)$ ) and outer London ( $45 \%$ (41\% to $48 \%))$. On $26 \%(21 \%$ to $32 \%)$ of wards, there were no nurses interacting with patients. A ward mean of 36\% (30\% to $41 \%$ ) of female patients had self contained, women-only facilities.
\end{abstract}

Conclusions Attention should focus on improving the quality of acute inpatient psychiatric care as well as of community care.

\section{Introduction}

Over the past 50 years the number of psychiatric inpatient beds in England has decreased greatly. The number has fallen from a peak of over 150000 in England in 1955 to 42000 in 1994-5. ${ }^{1}$ Despite the increase in community services, these beds still account for two thirds of expenditure on mental health services, ${ }^{2}$ and they remain an essential and large component of the range of services for severe mental illness. ${ }^{3}$ Despite the fall in overall bed numbers, the number of admissions a year has increased over the past decade from 207000 in 1984 to 237000 in 1994-5, representing an increase in throughput per bed each year, from 2.6 to 5.7 .

Acute inpatient services are reported to be under considerable pressure. ${ }^{4}$ The number of formal admissions increased by 53\% over five years, to 26100 in 1994-5. At the same time, some former inpatients on acute psychiatric wards have commented on the poor quality of care they received. ${ }^{6}$

On 21 November 1996 the Mental Health Act Commission, in collaboration with the Sainsbury Centre for Mental Health, made an unannounced visit to acute psychiatric wards in England and Wales. The innovative approach of a "national visit" was seen as a new and additional way for the commission to pursue its statutory responsibilities.

The aims of the national visit were identified by the commission in previous reports on matters of concern $^{78}$ : to investigate further the level of pressure on acute admission wards; to investigate the extent to which patients were going absent from the wards while still legally detained; to evaluate the level of nursing input to care; and to examine the appropriateness of ward facilities for the safe care of female patients. This paper reports how the visit was conducted and its findings.

\section{Methods}

\section{Sampling}

A list of all NHS trusts providing acute inpatient psychiatric care to people aged 16 to 64 years was obtained from the NHS year book $1995-6 .{ }^{9}$ Trusts were included if $(a)$ they had wards that were designated as acute; (b) most patients were in hospital for less than three months; and $(c)$ most patients were aged 18 to 65 . Wards designated as intensive care were included; medium secure units, regional secure units, special hospitals, the independent sector, and acute units providing only a "mother and baby" service were excluded. All the trusts on the list that were not known to the research team were telephoned so that details could be checked. Each of the Mental Health Act Commission's 